\title{
Kinematics and Mass Profile of AWM 7円
}

\author{
Daniel M. Koranyi and Margaret J. Geller \\ Harvard-Smithsonian Center for Astrophysics
}

\begin{abstract}
We have measured 492 redshifts (311 new) in the direction of the poor cluster AWM 7 and have identified 179 cluster members (73 new). We use two independent methods to derive a self-consistent mass profile, under the assumptions that the absorption-line galaxies are virialized and that they trace an underlying Navarro, Frenk \& White (1997) dark matter profile: (1) we fit such an NFW profile to the radial distribution of galaxy positions and to the velocity dispersion profile; (2) we apply the virial mass estimator to the cluster. With these assumptions, the two independent mass estimates agree to $\sim 15 \%$ within $1.7 h^{-1} \mathrm{Mpc}$, the radial extent of our data; we find an enclosed mass $\sim(3 \pm 0.5) \times 10^{14} h^{-1} \mathrm{M}_{\odot}$. The largest potential source of systematic error is the inclusion of young emission-line galaxies in the mass estimate.

We investigate the behavior of the surface term correction to the virial mass estimator under several assumptions about the velocity anisotropy profile, still within the context of the NFW model, and remark on the sensitivity of derived mass profiles to outliers. We find that one must have data out to a large radius in order to determine the mass robustly, and that the surface term correction is unreliable at small radii.
\end{abstract}

Subject headings: galaxies: clusters: individual (AWM 7) — galaxies: kinematics and dynamics

\section{Introduction}

The properties of galaxy clusters play an important role in constraining cosmological parameters and models of large-scale structure formation. Because clusters are the most massive collapsed aggregates in the universe, the cluster mass function and its evolution with redshift constrains the spectrum of density fluctuations (e.g. Eke, Cole \& Frenk 1996, Bahcall \& Cen 1992). Accurate cluster masses can also constrain the ratio of baryonic to total mass, and thus $\Omega_{0}$ (White et al. 1993).

\footnotetext{
1 Observations reported in this paper were obtained at the Multiple Mirror Telescope Observatory, a facility operated jointly by the University of Arizona and the Smithsonian Institution; at the Whipple Observatory, a facility operated jointly by the Smithsonian Astrophysical Observatory and Harvard University, and at the WIYN Observatory, a joint facility of the University of Wisconsin-Madison, Indiana University, Yale University, and the National Optical Astronomy Observatories.
} 
There are several standard ways of computing cluster masses. Optical redshift surveys yield a mass estimate through the application of the virial theorem, on the assumption that the galaxies trace the overall cluster mass distribution; this method was first applied to Coma by Zwicky (1933). More sophisticated variants of this method include a surface term to account for the unsampled portion of the cluster (The \& White 1986), and use the Jeans equation to account for the effects of orbital anisotropy (e.g. Binney \& Tremaine 1987). For clusters with many hundreds of redshifts, the distribution of infalling galaxies in redshift space measures the gravitational potential of the dark matter halo (Diaferio \& Geller 1997); this method has only been successfully applied to Coma so far (Geller, Diaferio \& Kurtz 1999). Observations of hot intracluster gas in the $\mathrm{X}$-ray provide information about the gravitational potential; the added assumption of hydrostatic equilibrium yields a mass (Bahcall \& Sarazin 1977). Gravitational lensing of background galaxies can also be used to estimate cluster masses (Webster 1985, Bartelmann et al. 1996 and references therein). Comparing cluster masses derived by these independent methods can provide insight into the internal structure of clusters (Loeb \& Mao 1994) and provides a consistency check on the mass derived by any one method.

While the distribution of total cluster masses constrains global cosmological parameters, knowledge of the density (or equivalently, mass) profiles of individual clusters constrains models of cluster formation. $N$-body simulations of hierarchical clustering by Navarro, Frenk \& White $(1995,1996,1997$, hereafter NFW) suggest that a universal two-parameter density model can adequately describe clusters over a broad range of masses. Until recently, few clusters had enough measured redshifts to constrain the mass profile; the advent of multi-fiber spectrographs has now made it possible to measure several hundred redshifts in a cluster within a reasonable time.

Our goal is to measure the mass profile of the cluster AWM 7, selected by Albert et al. (1977) on the basis of the cD galaxy at its center. Our sample consists of 179 cluster galaxies with measured redshifts; this work expands the sample of Koranyi et al. (1998) by $70 \%$, extending the surveyed region to a projected radius of $1.7 h^{-1} \mathrm{Mpc}$, and reaching fainter magnitudes in the core. We confirm the cold core seen in Koranyi et al. (1998), and fit a NFW mass profile from the projected distribution of radii for comparison with the virial mass profile; we consider also the effect of orbital anisotropies (still assuming the NFW profile) on the mass estimates. The two profiles agree remarkably well within our restricted context; the assumption of the NFW model produces self-consistent mass estimates using these two methods. Relaxing the assumption that the galaxies trace an underlying NFW dark matter distribution would result in a larger range of admissible masses (cf. The \& White 1986).

In $\S 2$ and $\S 3$, we describe the observations and data reduction. In $\S 4$ we describe the data, and in $\S 5$ we justify the choice of the NFW model, compute the resultant mass profiles, and comment on the reliability of the surface term correction. We defer discussion of some details until $\S 6$, and conclude in $\S 7$. We use $H_{0}=100 \mathrm{hm} \mathrm{s}^{-1} \mathrm{Mpc}^{-1}$ throughout. 


\section{Observations}

We observed a total of 593 distinct targets in the direction of AWM 7 from 1995 to 1999. We measured redshifts for 492 objects; 179 are cluster galaxies (with redshifts in the range 2500-7500 $\mathrm{km} \mathrm{s}^{-1}$ ), making AWM 7 one of the best-sampled clusters in the sky. Our sample extends over 1.5 degrees in radius from the cluster center, and reaches magnitudes as faint as $R=18$ in the core. We collected data from three different telescopes, with different sky coverage and different magnitude limits; we describe the details of each in turn. In each case we chose the spectral coverage so that both the Ca H-K break at $\lambda \lambda 3933,3968 \AA$ and $\mathrm{H} \alpha \lambda 6562 \AA$ would fall on the detector at the cluster redshift. These spectral features are important for determining redshifts by cross-correlation with template spectra. We list the redshifts for all measured cluster galaxies in Table 1 .

\subsection{FAST Observations}

We used the FAST Spectrograph (Fabricant et al. 1998) mounted on the 1.5-m Tillinghast telescope at the F.L. Whipple Observatory on Mt. Hopkins to observe 302 targets over 10 square degrees of sky centered on NGC 1129. We selected the targets from the Digitized Palomar Observatory Sky Survey scans. We used a 3 arcsecond slit and a 300 line $\mathrm{mm}^{-1}$ grating, resulting in a resolution of $\sim 6 \AA$ and spectral coverage of $3600-7600 \AA$. FAST can reliably measure redshifts for galaxies down to $R \sim 15.5$, and can measure redshifts for galaxies as faint as $R \sim 16$ if the central surface brightness is sufficiently high. 159 FAST targets are new to this paper; 51 of these are cluster members.

\subsection{MMT Observations}

We observed 45 targets with the Blue Channel spectrograph of the MMT on the nights of 1996 Dec. 5 and 6, using a 2 arcsecond slit and a 300 line $\mathrm{mm}^{-1}$ grating, resulting in a resolution of $\sim 10.6 \AA$ and spectral coverage of $3000-8000 \AA$. These targets are in the central $75^{\prime} \times 75^{\prime}$ $\left(1.15 \times 1.15 h^{-1} \mathrm{Mpc}\right)$ of the cluster, and are complete to $R=16.5$ in that region. These data have been previously published in Koranyi et al. (1998).

\subsection{Hydra Observations}

We observed 250 targets (under the queue observing scheme) with the Hydra multi-fiber bench spectrograph mounted on the WIYN telescope at Kitt Peak on the nights of 1998 Dec. 20 and 21 ; these data are new to this paper. There were 4 separate fiber configurations; 60 objects

were observed in more than one configuration. There were either four or five 25-minute exposures 
for each configuration. We used 3 arcsecond fibers, and a 400 line $\mathrm{mm}^{-1}$ grating, resulting in a resolution of $\sim 7 \AA$ and spectral coverage of 3800-7000 $\AA$. The four configurations were observed under strongly varying conditions, resulting in dramatic differences in throughput. Two of the configurations suffered from wind shake, and a third from cirrus. The remaining configuration was observed under stable, clear skies with good seeing and resulted in a redshift for almost every target.

In total, we were able to measure redshifts for 154 of the 250 targets (152 new), sometimes by combining spectra of the same object observed under different configurations. Only 22 of these are cluster members. The Hydra targets are all within the central $1.4^{\circ} \times 1.4^{\circ}$ of the cluster.

The magnitude completeness of the Hydra sample is complicated. Not only were we unable to measure a redshift for every target, but the original target list itself did not include every galaxy (up to some limit) in a magnitude-ordered list because of physical constraints in allocating fibers to objects. Moreover, the initial magnitude ranking was not by total galaxy luminosity, but rather by luminosity within the central 3 arcseconds subtended by the fibers. We will discuss the photometry and luminosity function of AWM 7 in a later paper; here, we are interested primarily in the kinematics of the cluster and the incompleteness is not a limitation on the analysis.

\section{Data Reduction}

We reduced all spectra using the xcsao task of the RVSAO package in IRAF (Kurtz \& Mink 1998). xcsao measures redshifts by cross-correlating the observed spectra against a suite of template spectra. The quality of the match is measured by the parameter $R$, an estimate of the ratio of the highest peak to the average noise peak in the correlation function. The template spectra are themselves empirically derived from an archive of FAST observations, and are thus strictly speaking directly applicable only to other FAST spectra. For the FAST velocities, the instrumental velocity offset with respect to the night sky lines is less than $10 \mathrm{~km} \mathrm{~s}^{-1}$ and is indistinguishable from zero (Kurtz \& Mink 1998). We have found that the FAST template spectra are reliable also for the MMT and Hydra spectra; for those objects observed with several spectrographs, the redshifts derived from the separate spectra all agree within the errors.

Many of the Hydra spectra have very low signal-to-noise ratios. We assess the robustness of the redshifts as follows: for each object, we construct all $2^{N}-1$ possible (non-zero) combinations of spectra from the $N$ individual exposures. $N$ is 4 or 5 for most objects, and 8,9 or 10 for objects observed in two configurations. For the few objects observed in 3 configurations, with $N$ as large as 13 , we examine only a subset of the 8191 possible combinations. We measure the redshift independently from each of these combined spectra. We accept a redshift for a target only if the following criteria all hold: (1) The $R$-value increases with the number of individual spectra contained in the combined spectrum; (2) the velocity of the combined spectra stabilizes as the number of constituent individual spectra increases; (3) the best combined spectrum has an 
$R$-value $>3$.

The comparatively narrow spectral range of the Hydra observations means that $\mathrm{H} \alpha$ is shifted off the edge of the detector for $c z \gtrsim 20000 \mathrm{~km} \mathrm{~s}^{-1}$. Cross-correlating emission line galaxies at these redshifts with the standard emission-line template results in a spurious redshift because the strongest remaining line in the spectrum is usually fit to $\mathrm{H} \alpha$. We solved this problem by constructing a new template that differs from the standard emission-line template only by the absence of the $\mathrm{H} \alpha$-nitrogen complex and the $\lambda \lambda 6717,6731 \AA$ sulfur lines.

We add an extra term of $65 \mathrm{~km} \mathrm{~s}^{-1}$ in quadrature to the errors of velocities determined from emission-line templates because the line emitting region may be offset in velocity from the systemic velocity of the galaxy (Kurtz \& Mink 1998).

Emission-line galaxies preferentially yield redshifts because an emission-line galaxy can be robustly matched to an emission template even when the signal-to-noise in the continuum is too low for a believable correlation with an absorption template. Thus, the completeness limit for emission-line galaxies is effectively fainter than for galaxies with no emission. The Hydra configuration observed through cirrus yielded few redshifts, resulting in a brighter effective magnitude limit; this effect is minor because there is considerable overlap on the sky among the four configurations.

\subsection{Spectral Classification}

We divide our spectra into two broad classes, emission (Em) and absorption (Ab), based on the presence or absence of $\mathrm{H} \alpha$ in the spectrum. $\mathrm{H} \alpha$ is "present" if its equivalent width

exceeds $5 \AA$. The robustness of this criterion is clearly a function of the signal-to-noise ratio of the spectrum; galaxies with low signal or with intrinsically weak $\mathrm{H} \alpha$ emission may be misclassified as absorption-line galaxies if the $\mathrm{H} \alpha$ peak does not rise appreciably above the noise. The misclassification is all in one direction: although some emission line galaxies may be misclassified as absorption, no absorption-line galaxy will be mistakenly classified as having emission.

\section{The Data}

Figure 1 (upper panel) shows the velocity distribution of all the observed galaxies. The cluster AWM 7, defined as in Koranyi et al. (1998) to consist of galaxies with redshifts in the range $2500-7500 \mathrm{~km} \mathrm{~s}^{-1}$, is cleanly and unambiguously distinguished from the background. The lower panel shows the velocity histogram of the cluster galaxies only; the solid histogram indicates the distribution of the entire sample, and the shaded histogram indicates the Em galaxies alone. Our cluster sample consists of 179 galaxies; we classify 42 as Em and 137 as Ab. A Kolmogorov-Smirnov test indicates that the probability of the Em and Ab samples being so 
discrepant if drawn from the same distribution is small $\left(P_{D>D_{\mathrm{obs}}}=0.0004\right)$; we conclude that the underlying distributions are different at the $\sim 4 \sigma$ confidence level.

We compute the mean velocity and dispersion for the different galaxy populations following the prescription of Danese, De Zotti, \& di Tullio (1980); the results are in Table 2. The Em galaxies have a much larger dispersion $\left(\sim 1100 \mathrm{~km} \mathrm{~s}^{-1}\right.$ vs. $\left.\sim 600 \mathrm{~km} \mathrm{~s}^{-1}\right)$, and are systematically offset in redshift by $\sim 400 \mathrm{~km} \mathrm{~s}^{-1}$ from the Ab population $\left(4800 \mathrm{~km} \mathrm{~s}^{-1} \mathrm{vs} .5200 \mathrm{~km} \mathrm{~s}^{-1}\right)$. The extrema of the Em galaxies' velocity histogram are almost equidistant from the Ab mean velocity, but near $3800 \mathrm{~km} \mathrm{~s}^{-1}$ there is an excess of Em galaxies above the otherwise almost uniform distribution; this excess decreases the mean redshift. Neither the $17 \mathrm{Em}$ galaxies with $3500<c z<4500 \mathrm{~km} \mathrm{~s}^{-1}$ nor the 12 with $3700<c z<4300 \mathrm{~km} \mathrm{~s}^{-1}$ evince any strong clustering on the sky. Absent independent distance measurements to these galaxies, it is difficult to determine whether they are foreground interlopers, or whether the cluster Em galaxy population is indeed offset in velocity.

The cD galaxy near the cluster center, NGC 1129, has a redshift of $5288 \pm 71 \mathrm{~km} \mathrm{~s}^{-1}$, which, within the errors, is at rest with respect to the absorption-line galaxy population. The peak of the ROSAT PSPC hard X-ray emission map, defined as the center of the pixel containing the greatest flux, is $6^{\prime \prime}$ from the nominal position of NGC 1129. We therefore adopt its position, $(\alpha, \delta)_{\mathrm{J} 2000}=(02: 54: 27.50,+41: 34: 42.50)$, as the center of the cluster.

Figure 2 shows the distribution of galaxies with measured redshifts on the sky. Open circles and filled triangles denote $\mathrm{Ab}$ and Em galaxies in the cluster, respectively; crosses denote foreground or background galaxies. We do not plot targets for which we were unable to measure a redshift. The enhancement in the surface number density near the center of the cluster is due to the fainter magnitude limit reached by the MMT and Hydra observations; most Hydra targets turned out to be background sources.

The Em galaxies are distributed much more uniformly on the sky than the Ab galaxies; the projected surface density of the Ab galaxies increases strongly toward the center, but the Em galaxies show no such enhancement. A two-dimensional Kolmogorov-Smirnov test (Press et al. 1992) indicates that the probability of drawing two such discrepant samples from the same underlying distribution is less than $5 \times 10^{-5}$. The distribution of Ab galaxies is strongly flattened; there is a pronounced elongation in the east-west direction.

The X-ray emission from AWM 7 is similarly elongated; Mohr et al. (1995) compute an axial ratio of $0.665 \pm 0.089$ at a position angle of $-83^{\circ} \pm 7^{\circ}$ from the X-ray flux within 7.5 of the cluster center, in perfect agreement with the value of 0.67 computed by Dell'Antonio et al. (1995) for an isothermal ellipsoid model density distribution. Mohr et al. (1995) report X-ray ellipticities for 58 clusters; AWM 7 is more flattened than both the mean and median clusters, but there are 10 clusters that are more flattened still. In our case it is difficult to measure the flattening quantitatively from our optical sample for comparison with the X-ray results because the completeness limit varies across our survey. 
Redshifts of cluster galaxies plotted against distance from the cluster center define a trumpet-shaped locus delimited by caustics (Kaiser 1987). Galaxies inside the caustics are bound to the cluster; those outside are unbound. We construct such a plot in Figure 3 (top panel); again, squares are Ab galaxies and triangles are Em galaxies. Although one can discern by eye where the caustics probably lie, they are in practice poorly defined. Their accurate determination requires many more galaxies than our sample contains (Diaferio 1999, Diaferio \& Geller 1997, Geller, Diaferio \& Kurtz 1999).

From the velocity histograms and the position-velocity diagrams it is clear that the Em and $\mathrm{Ab}$ galaxies are not equivalent tracers of the cluster mass. A friends-of-friends algorithm (Huchra \& Geller 1982) for finding substructure suggests that many of the Em galaxies are contained in smaller subgroups distinct from the main cluster (not shown); these may be accreting onto the cluster now, and, not yet being virialized, do not follow the dark matter distribution as closely as the $\mathrm{Ab}$ galaxies. There is considerable radial segregation of $\mathrm{Ab}$ and Em galaxies; the radial distribution of Em galaxies is more uniform and has a greater mean and median distance from the cluster center than that of the Ab galaxies. This behavior is observed in other clusters (Mohr et al. 1996, Adami et al. 1998), in groups (Mahdavi et al. 1999), and in the field as the morphology-density relation (Dressler 1980). Moreover, the velocity dispersion of the Em galaxies remains large with increasing radius while that of the Ab galaxies declines (see $\S 5$ ). These considerations suggest that the Em galaxies are not virialized tracers of the underlying dark matter distribution. Because our goal is an accurate determination of the mass profile, we exclude the Em galaxies from further analysis, following Mohr et al. (1996). We will revisit the issue of substructure in and around AWM 7 and other poor clusters in a subsequent paper.

Figure 3 (middle panel) shows the velocity dispersion profile constructed only from the Ab galaxies. We sort the galaxies by distance from the cluster center, then evaluate the velocity dispersion in a sliding bin containing 11 galaxies; we plot the dispersion as a function of the mean distance to the galaxies in the bin. Neighboring points are strongly correlated, as they share 10 of their 11 galaxies; every 11th point (distinguished by error bars) is independent.

Two large excursions from the overall trend dominate the profile. Two outlier galaxies, one at projected distance $R \sim 0.7 h^{-1} \mathrm{Mpc}$ and low velocity, the other at $R \sim 1.4 h^{-1} \mathrm{Mpc}$ and high velocity (circled in the top panel of the figure) produce these excursions. It is clear that these two galaxies are responsible because the number of data points in each excursion equals the width of the sliding bin used to compute the profile. We therefore define a subset of the Ab sample, $\mathrm{Ab}_{135}$, excluding these two galaxies. We shall henceforth refer to the entire sample of $137 \mathrm{Ab}$ galaxies as $\mathrm{Ab}_{137}$. The velocity dispersion profile for $\mathrm{Ab}_{135}$ appears in the lower panel; the overall dispersion of these 135 galaxies is $543_{-31}^{+37} \mathrm{~km} \mathrm{~s}^{-1}$ within $R=1.7 h^{-1}$ Mpc. After some fluctuations near the core, the profile becomes smooth and shows the characteristic declining profile seen in poor groups by Mahdavi et al. (1999) and in some $N$-body simulations (e.g. Crone, Evrard \& Richstone 1994). The decline in dispersion at small radii matches that of the "type C" clusters of Girardi et al. (1998). We revisit the issue of the cold core in $\S 6$. 


\section{Mass Profile}

We compute the mass profile of AWM 7 in two largely independent ways. First, we assume that the mass profile follows the functional form predicted by the hierarchical clustering simulations of Navarro, Frenk \& White $(1995,1996,1997)$. This model has two parameters which we derive separately from the observations under the assumption that the Ab galaxies are virialized and that they trace the dark matter distribution. While this is a restrictive assumption, there is growing evidence that the NFW profile is applicable beyond the hierarchical clustering regime in which it was proposed. Huss, Jain \& Steinmetz (1999) find from high-resolution $N$-body simulations that virialized dark matter halos with different formation histories nevertheless evince similar density and velocity dispersion profiles, and that this "universal" density profile is well described by the functional form of the NFW model. The collapse history of the cluster seems immaterial in determining the ultimate cluster profile. More concretely, Geller, Diaferio \& Kurtz (1999) show that this profile describes the Coma cluster well for $R \lesssim 10 h^{-1}$ Mpc. For comparison, we also fit a non-singular isothermal sphere to AWM 7.

We also compute the mass profile from the virial theorem alone. At small radii, this method gives incorrect results if not corrected for the exclusion of the outer cluster regions from the computation. The mass profile can be corrected with a surface term, which depends on both the mass and velocity dispersion profiles. These last two profiles are linked to the velocity anisotropy profile through the Jeans equation (e.g. Binney \& Tremaine 1987), but the data are insufficient

to constrain the problem completely. Usually one makes some assumptions about one or more of the profiles and solves for the others (e.g. Binney \& Mamon 1982, The \& White 1986, Binney \& Tremaine 1987, Girardi et al. 1998). Here, we make these assumptions only for the computation of the surface term correction to the virial mass estimator.

\subsection{The NFW model}

\subsubsection{Description}

The spherically symmetric NFW density distribution is

$$
\rho(r)=\frac{\delta_{c} \rho_{c}}{\left(r / r_{c}\right)\left(1+r / r_{c}\right)^{2}}
$$

resulting in the enclosed mass profile

$$
M(<r)=4 \pi \delta_{c} \rho_{c} r_{c}^{3}\left[\log \left(1+r / r_{c}\right)-\frac{r / r_{c}}{1+r / r_{c}}\right]
$$

where $\rho_{c}$ is the critical density of the universe, and $\delta_{c}$ and $r_{c}$ are the two parameters of the model, corresponding to the overall normalization and the core radius, respectively. The radial scale in the context of cluster simulations is often quoted in units of $r_{200}$, the radius where the mean 
cluster density has dropped to $200 \rho_{c}$. NFW claim, on the basis of numerical experiments, that this radius approximately separates the virialized and infall regions (see also Cole \& Lacey 1996). NFW define the "concentration parameter" $c=r_{200} / r_{c}$. A low value of $c$ arises if the core radius is comparable with the overall extent of the cluster, that is, the mass profile is not very concentrated toward the center. NFW demonstrate that more massive clusters are less concentrated, with the concentration parameter related to the overall normalization by

$$
\delta_{c}=\frac{200}{3} \frac{c^{3}}{\log (1+c)-c /(1+c)} .
$$

Because the enclosed mass of the NFW profile diverges logarithmically, it is customary to quote $M_{200} \equiv 200 \rho_{c}(4 \pi / 3) r_{200}^{3}$ as the cluster mass.

\subsubsection{Fitting the NFW Model}

We can derive $r_{c}$ observationally from the distribution of projected radii of cluster galaxies. The projected surface number density of galaxies in the NFW model is given by

$$
\Sigma(\tilde{R}) \propto \frac{1-X(\tilde{R})}{r_{c}^{2}\left(\tilde{R}^{2}-1\right)}
$$

where $\tilde{R}=R / r_{c}$ is the projected radius in units of the core radius, and

$$
X(\tilde{R})= \begin{cases}\left(1-\tilde{R}^{2}\right)^{-1 / 2} \operatorname{sech}^{-1} \tilde{R} & \text { if } \tilde{R}<1 \\ \left(\tilde{R}^{2}-1\right)^{-1 / 2} \sec ^{-1} \tilde{R} & \text { if } \tilde{R} \geq 1\end{cases}
$$

Given the distribution of projected radii in our sample, we fit $r_{c}$ with a maximum-likelihood technique by finding the value of $r_{c}$ that maximizes the probability

$$
\mathcal{L}=\prod_{i}\left[\frac{\tilde{R}_{i} \Sigma\left(\tilde{R}_{i}\right)}{\int_{0}^{\tilde{R}_{\max }\left(r_{c}\right)} \tilde{R} \Sigma(\tilde{R}) d \tilde{R}}\right]^{w_{i}}
$$

of observing that particular distribution of projected radii, where $w_{i}$ is a weight assigned to each galaxy to account for the non-uniform magnitude limit of our survey. We assign each galaxy one of two possible weights as follows: galaxies within the central $0.51 h^{-1} \mathrm{Mpc}$ of the survey, where it is $97 \%$ complete to $m_{R}=17.2$, have $w=1.00$; galaxies with $R_{i}>0.51 h^{-1} \mathrm{Mpc}$, where the survey is complete to $m_{R}=15.5$, have $w=1.95$, where 1.95 is the ratio of the number of galaxies with $m_{R}<17.2$ to the number with $m_{R}<15.5$ within $0.51 h^{-1} \mathrm{Mpc}$ of the cluster center. This correction assumes that the luminosity function is the same in the core as in the outskirts of the cluster. We exclude galaxies fainter than $m_{R}=17.2$ within $0.51 h^{-1} \mathrm{Mpc}$ and fainter than $m_{R}=15.5$ otherwise from the fit; there remain 53 galaxies with $R<0.51 h^{-1} \mathrm{Mpc}$ and 70 with $R \geq 0.51 h^{-1}$ Mpc. Both outliers remain in the sample. The denominator in Eq. 6 serves

to normalize the intrinsic probability of observing a particular galaxy $i$ at projected radius $\tilde{R}_{i}$ 
(expressed in the numerator) to the possible range of $\tilde{R}$ where it could be included in the sample. The upper limit of the integral is a function of $r_{c} ; \tilde{R}_{\max }\left(r_{c}\right)=R_{\lim } / r_{c}$, where $R_{\text {lim }}$ is the limiting projected radius of the survey. In practice, we use the largest projected radius in the sample for $R_{\text {lim. }}$. Table 3 lists the best-fit values of $r_{c}$ for the $\mathrm{Ab}_{135}$ and $\mathrm{Ab}_{137}$ subsamples, computed from 121 and 123 galaxies, respectively, along with 1-, 2-, and 3- $\sigma$ uncertainties, as determined by $\Delta(\log \mathcal{L})=0.5,1.0,1.5$. There is little difference in the best-fit $r_{c}$ for the two samples; if the Em galaxies are included, the best-fit $r_{c}$ increases by $\sim 50 \%$, because the Em galaxies are less centrally concentrated (Fig. 3). We compare the NFW model to the non-singular isothermal sphere in Section 5.2.

We use Monte Carlo simulations to assess the robustness of this method: for selected values of $r_{c}$, we construct 2000 sets of 123 projected radii drawn from the distribution of projected radii for an NFW profile with that $r_{c}$; we reproduce the non-uniform magnitude limit of our survey by only accepting galaxies with $R \geq 0.51 h^{-1} \mathrm{Mpc}$ with probability $1 / 1.95$. We compute the maximum-likelihood recovered $r_{c}$ using Eq. 6. Figure 1 shows the results for $r_{c}$ in the range $0.04-$ $0.30 h^{-1} \mathrm{Mpc}$. The median recovered $r_{c}$ is within 0.002 of the input $r_{c}$ in all but one case; however, the distributions of the recovered $r_{c}$ are broad. The distribution of recovered $r_{c}$ is narrowest for small $r_{c}$, and broadens as $r_{c}$ increases, because (1) the peak in the probability distribution of projected radii becomes less pronounced as $r_{c}$ increases, and because (2) we sample a smaller dynamic range of $\tilde{R}$ as $r_{c}$ increases (because the limiting projected radius $R_{\text {lim }}$ is constant). The distribution of the ratio of recovered to input $r_{c}$ is largely independent of input $r_{c}$, however. The lower and upper $1 \sigma$ bounds (defined as the values of $r_{c}$ enclosing $34.2 \%$ of the distribution on either side of the median) are typically displaced $-25 \%$ and $+30 \%$ from the true $r_{c}$, respectively. Increasing the sample size in the simulations to 500 radii (not shown) reduces the spread in the distribution of recovered $r_{c}$ by a factor of $\sim 2$.

Knowing $r_{c}$, the NFW model would be completely specified if we also knew $r_{200}$, but this quantity is difficult to derive observationally. We can arrive at an estimate if we assume that the virial theorem holds, so that $M_{v} \equiv 3 \sigma_{p}^{2} r_{v} G^{-1}$, where $G$ is the gravitational constant, $r_{v}$ is the virial radius, and $\sigma_{p}$ is the global line-of-sight cluster velocity dispersion. Then

$$
\frac{M_{200} r_{v}}{M_{v} r_{200}^{3}}=100 H_{0}^{2} \frac{(1+z)^{3}}{3 \sigma_{p}^{2}}
$$

where we have used $\rho_{c}(z)=3 H_{0}^{2}(1+z)^{3} /(8 \pi G)$ for $\Omega=1$, and the definition of $M_{200}$. The right-hand side of this equation is observationally determined, and substituting for $M_{v}$ and $M_{200}$ using Eq. 2, the left-hand side becomes a function of $r_{c}, r_{v}$, and $r_{200}$ which we solve for $r_{200}$. We fit $r_{c}$ from the distribution of projected radii, and approximate $r_{v}$ by

$$
r_{v} \sim \frac{\pi}{2} \frac{N(N-1)}{\Sigma_{i} \Sigma_{i<j} R_{i j}^{-1}}
$$

where $N$ is the number of galaxies in the system, and $R_{i j}$ is the projected separation between galaxies $i$ and $j$ (Binney \& Tremaine 1987). This estimator yields $r_{v} \sim 1.30 h^{-1} \mathrm{Mpc}$ for the $\mathrm{Ab}_{137}$ sample, and $r_{v} \sim 1.28 h^{-1} \mathrm{Mpc}$ for the $\mathrm{Ab}_{135}$ sample. 
The value of $r_{200}$ that solves Eq. 7 is fairly insensitive to the value of $r_{c}$; in the regime of interest, where $500<\sigma_{p}<600 \mathrm{~km} \mathrm{~s}^{-1}, r_{200}$ varies $\sim 3 \%$ as $r_{c}$ ranges from 0.12 to $0.30 \mathrm{~h}^{-1} \mathrm{Mpc}$ for any constant $\sigma_{p}$. The derived $r_{200}$ is more sensitive to $\sigma_{p}$, varying $\sim 15 \%$ over the range $500<\sigma_{p}<600 \mathrm{~km} \mathrm{~s}^{-1}$ with constant $r_{c}$. For the $r_{v}$ obtained from Eq. 8, the values of $r_{200}$ are 1.02 and $0.97 h^{-1} \mathrm{Mpc}$ for the $\mathrm{Ab}_{137}$ and $\mathrm{Ab}_{135}$ samples, respectively (Table 4 ). The corresponding value of the concentration parameter $c, 4.6$ in both cases, falls in the middle of the range 2.3-7.7 seen by Carlberg et al. (1997) for rich systems, although the errors are large in both cases. Comfortingly, the values are also smaller than the $c \sim 7.5$ that Mahdavi et al. (1999) find for their lower-mass poor groups. For AWM $7, M_{200}$ is 2.47 and $2.13 \times 10^{14} h^{-1} M_{\odot}$ for the $\mathrm{Ab}_{137}$ and $\mathrm{Ab}_{135}$ samples, respectively. Here the larger mass is associated with a formally slightly smaller value of $c$ (4.56 vs. 4.61), in keeping with the general trend seen by NFW, but this consistency may be accidental, considering the uncertainties in the computed values of $c$. It is interesting to note that two outliers (out of a sample of 137) can alter the recovered mass by $\sim 15 \%$.

Because the cluster extends beyond $r_{200}, M_{200}$ in fact underestimates the total mass. Extrapolating the NFW mass profile to $1.7 h^{-1} \mathrm{Mpc}$ (the maximum radial extent of the Ab sample) with Eq. 2 yields 3.49 and $3.01 \times 10^{14} h^{-1} M_{\odot}$ for the $\mathrm{Ab}_{137}$ and $\mathrm{Ab}_{135}$ samples, respectively.

\subsection{Comparison to the Non-Singular Isothermal Sphere}

The non-singular isothermal sphere (NSIS) arises from setting finite boundary conditions at zero radius to the same differential equation that gives rise to the singular isothermal sphere (e.g. Binney \& Tremaine 1987, §4.4). It is characterized by a density normalization $\rho_{0}$ and a core radius $r_{0}$ at which the the projected density falls to 0.5013 of its central value. In Fig. 5 we compare the fit of the projected surface number density from the NSIS and NFW models to the data. Although the maximum-likelihood technique of $\$ 5.1 .2$ makes optimal use of the data, it provides no independent assessment of goodness-of-fit. Here, although with some loss of information, we bin the observed surface number density for comparison with the models; we retain the same weighting scheme as in the maximum-likelihood calculation.

The left side of Fig. 5 shows the observed profile (points with Poisson error bars), the NFW fit (solid line), and the NSIS fit (dotted line) for both the $\mathrm{Ab}_{135}$ (top panel) and $\mathrm{Ab}_{135}$ (bottom) samples. The right side of the figure illustrates how the best-fit values of $r_{c}, r_{0}$, and $\chi^{2} / \nu$ vary with the choice of binning; the profiles and fits are shown for 11 galaxy equivalents per bin (galaxies beyond $0.51 h^{-1} \mathrm{Mpc}$ count as 1.95 equivalents).

For different binnings the quality of the fits of both the NFW and NSIS models, as measured by $\chi^{2} / \nu$, varies much less for the $\mathrm{Ab}_{135}$ sample than for the $\mathrm{Ab}_{137}$ sample. The best-fit values of $r_{c}$ and $r_{0}$ are quite stable for both samples. The decreased sensitivity to the binning provides further justification for excluding the two outlier galaxies. For both samples, the NFW model fits with a 
lower $\chi^{2} / \nu$ than the NSIS model, independent of the binning (except in one case). The superiority of the NFW model is more consistent for the $\mathrm{Ab}_{135}$ sample, although this superiority is not overwhelming; for $\mathrm{Ab}_{135}$, NFW fits with $\chi^{2} / \nu=1.075$, whereas the NSIS fits with $\chi^{2} / \nu=1.233$. For $\mathrm{Ab}_{137}$, the respective $\chi^{2} / \nu$ for NFW and NSIS are 1.184 and 1.252 (all for 11 galaxies per bin). There is thus some formal statistical justification for using the NFW model over the NSIS, but an unambiguous determination would require a survey out to 2 or $3 h^{-1} \mathrm{Mpc}$ (cf. Geller, Diaferio \& Kurtz 1999).

With 11 galaxies per bin, the best-fit values of $r_{c}$ are within $5 \%$ of the maximum-likelihood values for both samples, and well within the 1- $\sigma$ range for all binnings, indicating that the loss of information inherent in the binning is not severe.

\subsection{The Virial Mass Profile}

We now compute the mass profile of AWM 7 from the virial estimator appropriate for the case of galaxies embedded in a diffuse distribution of dark matter, under the added assumption that light traces mass. The appropriate estimator (Binney \& Tremaine 1987) is

$$
M_{\mathrm{est}}=\frac{3 \pi N}{2 G} \frac{\Sigma_{i} v_{i}^{2}}{\Sigma_{i} \Sigma_{j<i}\left|\mathbf{R}_{i}-\mathbf{R}_{j}\right|^{-1}}=\frac{3 r_{v}}{G} \frac{\Sigma_{i} v_{i}^{2}}{N-1}
$$

where $v_{i}$ is the radial velocity relative to the cluster mean, and $\mathbf{R}_{i}$ is the projected radius vector relative to the center of the cluster. This estimator assumes that the galaxies are in dynamical equilibrium within the cluster potential, that galaxies trace the dark matter distribution, and that the entire cluster has been observed. These assumptions do not apply to the Em galaxy population; indeed, when we apply this prescription to the Em galaxies alone, the resultant mass estimate is a factor of $\sim 4$ greater than for the $\mathrm{Ab}$ galaxies. To correct for the failure to include

the entire angular extent of the cluster in our sample, we correct the mass estimate with a surface term $C(b)$ (The \& White 1986) that depends on the limiting radius $b$ of the observations. The corrected mass is

$$
M_{c v}(<b)=M_{\mathrm{est}}[1-C(b)]=M_{\mathrm{est}}\left[1-4 \pi b^{3} \frac{\rho(b)}{\int_{0}^{b} 4 \pi r^{2} \rho(r) d r} \frac{\sigma_{r}^{2}(b)}{\sigma^{2}(<b)}\right]
$$

where $\sigma(<b)$ denotes the integrated velocity dispersion within the limiting radius $b$ (e.g. Girardi et al. 1998).

The surface term incorporates information about the velocity dispersion profile and the density profile; the former is measurable from the data, but some assumption must be made about the form of the density profile - in order to correct that very profile. The \& White (1986) and Girardi et al. (1998) posit a functional form for the mass profile, which they then fit to the data on the basis of the projected number density of galaxies. Here we assume an underlying NFW mass profile. 


\subsubsection{Behavior of The Surface Term}

The radial dependence in the NFW model of the surface term in Eq. 10 is

$$
C_{\mathrm{NFW}}(r)=\frac{u^{2}}{(1+u)^{2}}\left[\log (1+u)-\frac{u}{1+u}\right]^{-1}\left[\frac{\sigma_{r}(r)}{\sigma(<r)}\right]^{2}
$$

where $u=r / r_{c}$. The last term involves both the overall velocity dispersion $\sigma$ and the radial velocity dispersion $\sigma_{r}$, and is thus a function of the anisotropy parameter $\beta=1-\sigma_{\theta}^{2} / \sigma_{r}^{2}$, which is itself a function of $r$. Now $\sigma^{2}=\sigma_{r}^{2}+\sigma_{\theta}^{2}+\sigma_{\phi}^{2}$; under the assumption of spherical symmetry the last two terms are equal, so $\sigma^{2}=(3-2 \beta) \sigma_{r}^{2}$, which we substitute into the denominator of the last term for computation. The anisotropy and velocity dispersion profiles are not independent; they are coupled via the Jeans equation. Girardi et al. (1998) find that for their mass model a constant anisotropy profile $\beta(r)=0$ produces a velocity dispersion profile that peaks at $\sim 0.1 r_{v}$, dips slightly in the core, and decreases gradually at large radii; the simple functional form $\beta(r)=-k / r$, where $k$ is a constant, produces a velocity dispersion profile with a more severe dip in the core. In this case, the orbits in the core become purely tangential as $\beta \rightarrow-\infty$.

Figure 6 shows the behavior of the surface term for an NFW mass distribution and the observed velocity dispersion profile (Fig. 3) under the assumption of three different anisotropy profiles: constant (top panel), $\beta(r)=-k / r$ (middle), and $\beta(r)=1-k / r$ (bottom). The multiple curves in each panel correspond to the values of $\beta$ (top) or $k$ (middle and bottom) enclosed in brackets. One of the extreme curves is labeled in each case for reference. The values of $k$ are chosen to bracket the value inferred from the profiles in Fig. 3 of Girardi et al. (1998). Note that the three panels are not drawn to the same scale. Because these classes of $\beta$ profile are derived from fits to the mass model of Girardi et al. (1998), they are not a priori strictly valid for the NFW profile; nevertheless, it is reasonable to adopt them here as representative of the general forms that the profiles may have.

By definition, the surface term must be everywhere less than unity (to ensure a positive mass). The results shown for constant large values of $\beta$ are thus clearly unphysical. At small radii, the data are too noisy for all our assumptions to hold; a proper treatment would require a full solution of the Jeans equation. It is unlikely that this treatment would improve the results very much, however, because of the intrinsic noisiness and discreteness of the data. At larger radii, the variations in the surface term become less pronounced, and the surface term in general becomes less important, decreasing to $\lesssim 5 \%$ for each profile at the outer limit of our sample. Sufficiently far from the core, then, the virial mass estimator provides a robust estimation of the enclosed cluster mass, but at small radii the surface correction is too variable to result in a reliable mass profile. The virial mass estimator thus seems to be a good tool for estimating total cluster masses, but it is not well suited to the determination of mass profiles in cluster cores, where the surface term correction is most important but least well determined.

A reliable description of the surface term at small radii may only be possible from cluster simulations in which the dark matter density and velocity distributions are fully known to the 
experimenter. In that case, empirical surface term profiles can be built from the data and compared to reconstructions that an observer would generate under various assumptions of the anisotropy profile.

\subsubsection{The Virial Mass Profile}

Figure 7 shows the virial mass profile (Eq. 9), with no surface term correction, as derived from three subsets of our galaxy sample: the entire sample (crosses), the $\mathrm{Ab}_{137}$ sample (triangles), and the $\mathrm{Ab}_{135}$ sample (squares). The entire (Em+Ab) sample is included only to illustrate that the inclusion of the emission-line galaxies with their larger velocity dispersion skews the results towards higher mass. Indeed, this difference is evident in the two Ab profiles as well: they agree up to the first outlier, at which point the $\mathrm{Ab}_{137}$ mass profile jumps; the two Ab profiles then grow more or less in parallel until the next outlier. We compute uncorrected enclosed masses within 1.7 $h^{-1} \mathrm{Mpc}$ of 3.10 and $2.74 \times 10^{14} h^{-1} M_{\odot}$ from the $\mathrm{Ab}_{137}$ and $\mathrm{Ab}_{135}$ samples, respectively. The surface term correction at this radius is on the order of $2-5 \%$, depending on the anisotropy.

We estimate the error in the enclosed mass profile by the statistical bootstrap method (Diaconis \& Efron 1983): for the $N$ galaxies enclosed within a given projected radius, we compute the $N$ possible enclosed masses from all subsets of $N-1$ galaxies. We take the standard deviation of the resultant set of masses as the formal error in the estimated enclosed mass. The formal errors become quite small, on the order of $3 \%$, at large radii. When the number of galaxies is large and the distribution of velocities is well-behaved, the random rearrangement of velocities has little effect on the mass estimate; thus, the distribution of the bootstrapped masses is narrow. The actual error is in fact larger, as demonstrated by the sensitivity to the outliers. The error in the enclosed virial mass is $\sim 15 \%$.

The solid smooth curves indicate the NFW mass profiles we fit previously, and the dotted lines indicate the profiles corresponding to the $1 \sigma$ ranges of $r_{c}$ from Table 3 with $r_{200}$ recomputed from Eq. 7. The agreement with the virial mass is quite good, to within $\sim 15 \%$ at $1.7 h^{-1} \mathrm{Mpc}$ for both Ab samples, with the virial mass the smaller of the two in each case. The agreement along the profile in general is reasonable given the uncertainty in the surface term. The discrepancy is greatest near $R \sim 0.5 h^{-1} \mathrm{Mpc}$, corresponding to $r / r_{c} \sim 2$, where the surface term correction can be on the order of $20 \%$ in the proper direction; at the virial radius the masses are in perfect agreement, and at larger radii the NFW mass profiles overtake the virial mass profiles. Because $r_{200}$ is insensitive to $r_{c}$, the variation in $r_{c}$ results in little difference in the mass profile at large radii, but of course has a greater effect on scales of a few times $r_{c}$. Table 5 compares the NFW and virial masses. Based on the sensitivity of the virial mass to the outliers and on the effect of the surface term, we assign an error of $0.5 \times 10^{14} h^{-1} \mathrm{M}_{\odot}$ to our mass estimate.

Our mass profile is in agreement with the ROSAT X-ray mass of Dell'Antonio et al. (1995),

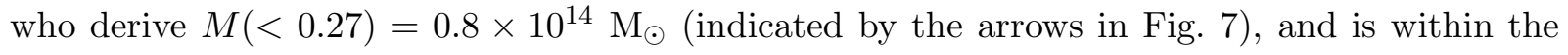


allowable range of mass profiles determined from ROSAT data by Neumann \& Böhringer (1995) and from ASCA data by Loewenstein \& Mushotzky (1996) and Markevitch \& Vikhlinin (1997). The X-ray masses are all quite uncertain and extend only to $R \sim 0.5 h^{-1}$ Mpc. Koranyi et al. (1998) illustrate the range of mass profiles from X-ray determinations; the range is so broad as to include even the virial mass profile computed from emission-line galaxies; any plausible kinematic mass profile is thus likely to be consistent with the current X-ray limits on the mass. The increased sensitivity of the Chandra Observatory will allow X-ray mass profiles to be determined with more precision and to larger radii.

\section{Discussion}

Galaxy clusters display a range of velocity dispersion profiles ( e.g. den Hartog \& Katgert 1996, Girardi et al. 1998). Some are peaked at the center, others are more or less flat, still others rise with increasing radius near the center. This variety suggests that clusters today are still in a variety of dynamical states, and that indiscriminately combining a set of clusters to improve the statistics can easily lead to incorrect results if clusters with different kinematics are averaged together. Even within a single cluster, it is important to discriminate between the generally red, virialized population and the newly infalling bluer galaxies; including the latter in steady-state kinematical analyses will artificially inflate the mass estimate; we suggest that this effect accounts for the mass of $5.77_{-1.50}^{+1.80} h^{-1} M_{\odot}$ derived for AWM 7 by Girardi et al. (1998).

In principle, the non-virialized Em galaxies can be used as tracers of the escape velocity to arrive at an independent mass measurement (Diaferio \& Geller 1997, Diaferio 1999). In practice, there are not enough Em galaxies in our sample for this method to yield a robust mass estimate; although the formal mass returned by this method for AWM 7 (kindly computed for us by A. Diaferio) is comparable to the ones we derive here, the fractional error in that mass is $\sim 0.9$.

Our enlarged sample confirms the presence of a cold core in the cluster (Koranyi et al. 1998); although still noisy near the cluster center, the velocity dispersion within a projected radius of $\sim 0.2 h^{-1} \mathrm{Mpc}$ is lower than in the range $0.2-0.5 h^{-1} \mathrm{Mpc}$. Beyond $\sim 1 h^{-1} \mathrm{Mpc}$ the velocity dispersion drops below the range in the core. Abell 576 (Mohr et al. 1996), a similarly well-sampled cluster, has a more pronounced cold core but a rising absorption-line velocity dispersion profile in the range 0.4-1.2 $h^{-1}$ Mpc. A simple dynamical argument suggests that cold cores arise naturally from the NFW density profile. Assuming virial equilibrium to hold in the core, $\sigma^{2} \propto G M(<r) / r$. Modeling the density profile at small radii as a power law $\rho(r) \propto r^{-\alpha}$ yields $M(<r) \propto r^{3-\alpha}$, so $\sigma \propto r^{1-\alpha / 2}$. For the NFW profile $\alpha=1$ in the core, resulting in a rising velocity dispersion profile.

Because the velocity dispersion profile, mass profile, and orbital anisotropy profile are coupled through the Jeans equation, it is difficult to derive a mass profile without making some assumptions about the form of one or more of these functions along the way. The projected velocity dispersion profile is directly observable, but it is sensitive to outliers and substructure, and 
requires either extensive smoothing or a large number of galaxies for a robust determination. The anisotropy profile is also difficult to constrain, with some evidence for a variety of profiles possible in clusters (Girardi et al. 1998). The mass profile can be estimated from X-ray observations, but these tend to have few data points, large errors, and cover only the inner regions of clusters; consequently, they can be fit by a wide range of models, resulting in large uncertainties in mass when extrapolated to large cluster radii.

It seems difficult to avoid circular logic in computing the surface term, as it depends on both the anisotropy profile and the density distribution that one is trying to determine. One can make a self-consistency argument, as we do here, to verify that the assumed density profile used to correct the virial mass profile agrees with the virial profile so corrected; if the surface term were small, this circularity would be less troublesome, because the assumed density profile would affect the virial mass profile only through that small correction. But as Fig. 6 illustrates, the surface term correction is often significant, particularly at small radii. Not only is the correction large, but it is also uncertain, sensitive to uncertainty in the anisotropy profile alone. The coupling between the mass profile and the surface term is thus quite strong; even with the assumption of a density profile (which in principle renders the whole exercise redundant because the mass profile is then already known), the surface term correction is too poorly constrained to produce a reliable mass profile. Only at the edges of the cluster does the surface term correction become reliable, but by then it is no longer very important. Accurate determination of cluster masses from the virial estimator, then, requires broad angular coverage; dense sampling of the core is not enough.

\section{Conclusion}

We derive the mass profile of AWM 7 in two independent ways from an optical sample of 179 galaxies with redshifts within $1.7 \mathrm{~h}^{-1} \mathrm{Mpc}$ of the cluster center; we use only the 137 absorption-line galaxies (and a 135-element subset of them) in the analysis. One method uses the surface number density and global velocity dispersion; the other uses individual velocities and pairwise distances. Both assume and underlying NFW profile. The two methods yield remarkably consistent mass estimates, particularly at the outer limit of our sample; the enclosed mass within a projected radius of $1.7 h^{-1} \mathrm{Mpc}$ agrees within $15 \%$ for both samples. The mass estimates are sensitive to the inclusion of two outliers in the sample; both the NFW and virial masses increase by $\sim 15 \%$ when they are included. Including the Em galaxies, with their larger dispersion, in the calculation results in a virial mass larger by a factor of two. The cluster mass within $1.7 h^{-1} \mathrm{Mpc}$ is $\sim(3 \pm 0.5) \times 10^{14} h^{-1} \mathrm{M}_{\odot}$.

The surface term correction to the virial mass estimator is sensitive to noise in the velocity dispersion profile and to the assumed velocity anisotropy profile. The surface term at small radii is too unreliable for the virial mass estimator to be used for measuring mass profiles; at large radii, where the surface term is more certain, it is no longer an important correction to the enclosed mass. The virial mass estimator is thus reliable for measuring total cluster masses, but 
not enclosed mass profiles; to overcome the limitations of the surface term at small radii, cluster redshift samples must have broad angular coverage. Dense sampling of cluster cores can reduce the scatter in the surface term if there are enough galaxies in the core, but our experience with AWM 7 (22 cluster members out of 154 Hydra redshifts) suggests that telescope time is better spent probing the outskirts of the cluster.

Untangling the relation between the anisotropy profile, velocity dispersion profile, and density profile in cluster cores may ultimately require high-resolution simulations that track both dark matter particles and, once they have formed, galaxies.

We thank Jim Peters, Perry Berlind, and Mike Calkins for obtaining many of the FAST spectra reported herein, and Susan Tokarz for preliminary reductions. We thank Mike Kurtz and Doug Mink for advice in reducing the Hydra spectra, Norman Grogin and Andisheh Mahdavi for useful discussions, and Antonaldo Diaferio for lucid discussions and for running our data through his caustic-finding algorithm.

This work is supported by the Smithsonian Institution.

\section{REFERENCES}

Adami, C., Biviano, A. \& Mazure, A., 1998, A\&A, 331, 439

Albert, C., White, R. \& Morgan, W., 1977, ApJ, 211, 309

Bahcall, J.N. \& Sarazin, C.L., 1977, ApJ, 213, L99

Bahcall, J.N. \& Cen, R.Y., 1992, ApJ, 398, L81

Bartelmann, M., Narayan, R., Seitz, S. \& Schneider, P., 1996, ApJ, 464, L115

Binney, J. \& Mamon, G.A., 1982, MNRAS, 200, 361

Binney, J. \& Tremaine, S., 1987, Galactic Dynamics. Princeton University Press

Carlberg, R.G., Yee, H.K.C., Ellingson, E., Morris, S.L., Abraham, R., Gravel, P., Pritchet, C.J., Smecker-Hane, T., Hartwick, F.D.A., Hessler, J.E., Hutchings, J.B. \& Oke, J.B., 1997, ApJ, 476, L7

Cole, S. \& Lacey, C., 1996, MNRAS, 281, 716

Crone, M.M., Evrard, A.E. \& Richstone, D.O., 1994, ApJ, 434, 402

Danese, L., De Zotti, G. \& di Tullio, G., 1980, A\&A, 82, 322

Dell'Antonio, I.P., Geller, M.J. \& Fabricant, D.G., 1995, AJ, 110, 502

den Hartog, R. \& Katgert, P., 1996, MNRAS, 279, 349

Diaconis, P. \& Efron, B., 1983, Sci. Am., 248, 116

Diaferio, A. \& Geller, M.J., 1997, ApJ, 481, 663 
Diaferio, A., 1999, MNRAS, in press, astro-ph/9906331

Dressler, A., 1980, ApJ, 236, 351

Eke, V.R., Cole, S. \& Frenk, C.S., 1996 MNRAS, 282, 263

Fabricant, D.G., Cheimets, P., Caldwell, N. \& Geary, J., 1998, PASP, 110, 79

Geller, M.J., Diaferio, A. \& Kurtz, M.J., 1999, ApJ, 517, L23

Girardi, M., Giuricin, G., Mardirossian, F., Mezzetti, M. \& Boschin, M., 1998, ApJ, 505, 74

Huchra, J.P. \& Geller, M.J., 1982 ApJ, 257, 423

Huss, A., Jain, B. \& Steinmetz, M., 1999 ApJ, 517, 64

Kaiser, N., 1987, MNRAS, 277, 1

Kurtz, M.J. \& Mink, D., 1998, PASP, 110, 934

Koranyi, D.M., Geller, M.J., Mohr, J.J. \& Wegner, G., 1998, AJ, 116, 2108

Loeb, A. \& Mao, S., 1994, ApJ, 435, L109

Loewenstein, M. \& Mushotzky, R.F., 1996, ApJ, 471, L83

Mahdavi, A., Geller, M.J., Böhringer, H., Kurtz, M.J. \& Ramella, M., 1999, ApJ, 518, 69

Markevitch, M. \& Vikhlinin, A., 1997, ApJ, 474, 84

Mohr, J.J., Evrard, A.E., Fabricant, D.G. \& Geller, M.J., 1995, ApJ, 447, 8

Mohr, J.J., Geller, M.J., Fabricant, D.G., Wegner, G., Thorstensen, J. \& Richstone, D.O., 1996, ApJ, 470, 724

Navarro, J.F., Frenk, C.S. \& White, S.D.M., 1995, MNRAS, 275, 720

Navarro, J.F., Frenk, C.S. \& White, S.D.M., 1996, ApJ, 462, 563

Navarro, J.F., Frenk, C.S. \& White, S.D.M., 1997, ApJ, 490, 493

Neumann, D.M. \& Böhringer, H., 1995, A\&A, 301, 865 (NB)

Press, W.H., Flannery, B.P., Teukolsky, S.A. \& Vetterling, W.T., 1992, Numerical Recipes: The Art of Scientific Computing 2nd ed. Cambridge University Press

The, L.S. \& White, S.D.M., 1986, AJ, 92, 1248

Webster, R.L., 1985, MNRAS, 213, 871

White, S.D.M., Navarro, J.F., Evrard, A.E. \& Frenk, C.S., 1993, Nature, 366, 429

Zwicky, F., 1933, Helv. Phys. Acta, 6, 110

This preprint was prepared with the AAS LATEX macros v4.0. 
Table 1. Redshifts of Target Galaxies

\begin{tabular}{|c|c|c|c|c|c|c|c|}
\hline$\alpha_{2000}$ & $\delta_{2000}$ & $\begin{array}{c}c z \\
{\left[\mathrm{~km} \mathrm{~s}^{-1}\right]}\end{array}$ & $\begin{array}{c}\sigma_{c z} \\
{\left[\mathrm{~km} \mathrm{~s}^{-1}\right]}\end{array}$ & $\alpha_{2000}$ & $\delta_{2000}$ & $\begin{array}{c}c z \\
{\left[\mathrm{~km} \mathrm{~s}^{-1}\right]}\end{array}$ & $\begin{array}{c}\sigma_{c z} \\
{\left[\mathrm{~km} \mathrm{~s}^{-1}\right]}\end{array}$ \\
\hline 24512.81 & 414404.57 & 4970 & 49 & 25444.00 & 413918.00 & 5372 & 25 \\
\hline 24531.90 & 411434.34 & 5127 & 51 & 25444.10 & 415208.80 & 5974 & 24 \\
\hline 24536.34 & 420927.32 & 5063 & 22 & 25444.60 & 413141.40 & 4450 & 22 \\
\hline 24559.29 & 411918.70 & 5316 & 19 & 25447.60 & 411850.00 & 5904 & 40 \\
\hline 24624.61 & 411636.62 & 4672 & 34 & 25448.10 & 412434.00 & 5622 & 16 \\
\hline 24657.12 & 415915.58 & 5098 & 38 & 25449.80 & 413725.39 & 5994 & 38 \\
\hline 24658.43 & 414633.28 & 4628 & 23 & 25455.30 & 412415.41 & 5423 & 42 \\
\hline 24740.54 & 402941.96 & 5607 & 32 & 25456.30 & 412946.79 & 4615 & 41 \\
\hline 24757.73 & 401829.92 & 4985 & 22 & 25500.60 & 420832.00 & 5613 & 26 \\
\hline 24758.14 & 411923.95 & 4855 & 47 & 25501.80 & 412651.30 & 4918 & 51 \\
\hline 24817.12 & 410949.21 & 5090 & 30 & 25502.00 & 413130.40 & 5781 & 22 \\
\hline 24822.03 & 413647.23 & 5217 & 23 & 25502.13 & 410349.45 & 4556 & 41 \\
\hline 24849.65 & 404046.42 & 5219 & 19 & 25502.40 & 413624.10 & 4175 & 18 \\
\hline 24859.01 & 410221.12 & 5169 & 43 & 25504.80 & $42 \quad 1545.80$ & 5223 & 23 \\
\hline 24909.34 & 413457.79 & 4686 & 24 & 25505.53 & 413828.90 & 5377 & 32 \\
\hline 24909.55 & 413447.71 & 4698 & 22 & 25506.00 & 414400.38 & 5926 & 53 \\
\hline 24920.60 & 405317.02 & 4160 & 31 & 25508.20 & 412917.09 & 4917 & 40 \\
\hline 24940.90 & 410317.00 & 5488 & 31 & 25514.10 & 414729.80 & 5797 & 52 \\
\hline 24948.10 & 412745.50 & 5279 & 17 & 25516.60 & 412020.51 & 5324 & 31 \\
\hline 24951.00 & 413403.70 & 5999 & 29 & 25517.33 & 413449.01 & 5177 & 25 \\
\hline 25003.48 & 430009.54 & 5052 & 45 & 25519.01 & 413428.02 & 5411 & 21 \\
\hline 25052.80 & 414552.70 & 5449 & 62 & 25520.97 & 413833.90 & 4742 & 38 \\
\hline 25117.70 & 410918.40 & 3433 & 21 & 25528.58 & 414440.95 & 5685 & 18 \\
\hline 25118.60 & 413355.10 & 5733 & 34 & 25532.50 & 412953.30 & 5735 & 49 \\
\hline 25143.22 & 424943.39 & 5420 & 33 & 25538.48 & 412758.40 & 5600 & 63 \\
\hline 25152.26 & 413038.88 & 4324 & 68 & 25543.55 & 412837.70 & 5538 & 66 \\
\hline 25207.90 & 413445.90 & 4572 & 17 & 25544.03 & 410748.05 & 5559 & 17 \\
\hline 25215.50 & 412355.10 & 5835 & 24 & 25548.00 & 415920.11 & 4423 & 23 \\
\hline 25233.87 & 412148.90 & 5340 & 45 & 25551.80 & 413239.01 & 4224 & 77 \\
\hline 25238.50 & 413441.10 & 4618 & 20 & 25552.80 & 404905.59 & 5727 & 31 \\
\hline 25242.30 & 411933.50 & 4471 & 54 & 25554.00 & 414437.60 & 4317 & 94 \\
\hline 25243.10 & 410608.76 & 5957 & 32 & 25555.30 & 413458.10 & 4748 & 27 \\
\hline 25251.95 & 412621.30 & 5725 & 73 & 25559.20 & 413610.40 & 5957 & 67 \\
\hline 25258.90 & 413201.60 & 6770 & 25 & 25604.61 & 413808.74 & 4466 & 29 \\
\hline 25259.00 & 415824.71 & 5387 & 26 & 25607.40 & 413751.10 & 5948 & 26 \\
\hline 25305.61 & 410427.94 & 4936 & 24 & 25614.82 & 413850.80 & 5234 & 59 \\
\hline
\end{tabular}


Table 1-Continued

\begin{tabular}{|c|c|c|c|c|c|c|c|}
\hline$\alpha_{2000}$ & $\delta_{2000}$ & $\begin{array}{c}c z \\
{\left[\mathrm{~km} \mathrm{~s}^{-1}\right]}\end{array}$ & $\begin{array}{c}\sigma_{c z} \\
{\left[\mathrm{~km} \mathrm{~s}^{-1}\right]}\end{array}$ & $\alpha_{2000}$ & $\delta_{2000}$ & $\begin{array}{c}c z \\
{\left[\mathrm{~km} \mathrm{~s}^{-1}\right]}\end{array}$ & $\begin{array}{c}\sigma_{c z} \\
{\left[\mathrm{~km} \mathrm{~s}^{-1}\right]}\end{array}$ \\
\hline 25307.70 & 412503.68 & 5613 & 32 & 25626.70 & 410759.09 & 5092 & 41 \\
\hline 25316.20 & 412911.80 & 5262 & 26 & 25630.10 & 421635.90 & 5882 & 53 \\
\hline 25320.75 & 415648.30 & 5440 & 37 & 25632.60 & 410044.30 & 5596 & 19 \\
\hline 25326.28 & 415635.20 & 5338 & 44 & 25638.60 & 411959.82 & 4633 & 10 \\
\hline 25330.00 & 413903.38 & 4840 & 32 & 25653.90 & 413933.19 & 5374 & 45 \\
\hline 25340.20 & 414331.10 & 6290 & 28 & 25654.72 & 412343.30 & 5594 & 60 \\
\hline 25344.10 & 412715.08 & 4010 & 32 & 25656.03 & 412049.54 & 5153 & 41 \\
\hline 25347.10 & 413249.09 & 5161 & 31 & 25656.20 & 415841.90 & 5331 & 17 \\
\hline 25350.00 & 412718.40 & 4616 & 44 & 25708.97 & 413100.20 & 6334 & 31 \\
\hline 25351.30 & 414338.20 & 5256 & 18 & 25722.00 & 415619.50 & 4439 & 20 \\
\hline 25354.50 & 414025.61 & 5561 & 49 & 25730.97 & 413749.31 & 4498 & 42 \\
\hline 25359.50 & 414735.09 & 6324 & 23 & 25733.50 & 413057.90 & 4943 & 21 \\
\hline 25403.46 & 413329.00 & 6107 & 50 & 25736.79 & 413256.89 & 5121 & 23 \\
\hline 25405.72 & 415744.90 & 4331 & 43 & 25757.22 & 424605.92 & 5338 & 35 \\
\hline 25414.80 & 412300.00 & 6413 & 17 & 25811.85 & 425527.98 & 5417 & 44 \\
\hline 25416.40 & 413938.41 & 5150 & 30 & 25812.40 & 414211.90 & 5658 & 26 \\
\hline 25416.98 & 413452.30 & 5477 & 55 & 25858.80 & 411717.50 & 5065 & 25 \\
\hline 25424.40 & 413619.40 & 6129 & 26 & 25901.10 & 422045.50 & 4954 & 22 \\
\hline 25425.30 & 413435.90 & 5085 & 17 & 25932.20 & 412232.80 & 5753 & 20 \\
\hline 25426.85 & 413919.33 & 5700 & 11 & 25941.10 & 413454.80 & 4956 & 29 \\
\hline 25427.40 & 413047.30 & 4827 & 73 & 25953.36 & 413243.84 & 5625 & 23 \\
\hline 25427.50 & 413442.50 & 5288 & 71 & 30040.99 & 422206.64 & 6013 & 25 \\
\hline 25428.56 & 413558.00 & 5272 & 41 & 30054.87 & 414221.02 & 4911 & 32 \\
\hline 25428.57 & 412655.94 & 4905 & 23 & 30058.05 & 430049.90 & 5025 & 25 \\
\hline 25429.04 & 411007.60 & 4528 & 39 & 30059.45 & 430103.50 & 4828 & 22 \\
\hline 25430.40 & 413636.22 & 6230 & 24 & 30219.90 & 405400.31 & 4703 & 98 \\
\hline 25434.01 & 413331.27 & 5351 & 17 & 30220.07 & 415905.42 & 6736 & 25 \\
\hline 25436.00 & 415325.00 & 5407 & 22 & 30244.32 & 413730.65 & 4976 & 30 \\
\hline 25438.30 & 413518.10 & 5645 & 26 & $\begin{array}{lll}302 & 49.28\end{array}$ & 405815.97 & 4851 & 54 \\
\hline 25439.25 & 420936.93 & 6101 & 16 & 30330.86 & 413623.34 & 4797 & 28 \\
\hline 25440.00 & 413431.12 & 5006 & 39 & 30336.80 & 410956.78 & 4871 & 21 \\
\hline 25441.40 & 413349.28 & 5291 & 45 & & & 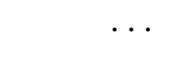 & $\ldots$ \\
\hline
\end{tabular}

Note. - RA and Dec (J2000), and redshift and error (in $\mathrm{km} \mathrm{s}^{-1}$ ) for galaxies with measured redshifts. 
Table 2. Velocity Distribution

\begin{tabular}{lrrrrr}
\hline \hline Sample & $\mathrm{N}_{\text {gal }}$ & \multicolumn{1}{c}{$\overline{c z}$} & $\begin{array}{c}\sigma_{\overline{c z}} \\
{\left[\mathrm{~km} \mathrm{~s}^{-1}\right]}\end{array}$ & $\begin{array}{c}\sigma_{p} \\
{\left[\mathrm{~km} \mathrm{~s}^{-1}\right]}\end{array}$ & \multicolumn{1}{c}{$\begin{array}{c}\sigma_{200} \\
{\left[\mathrm{~km} \mathrm{~s}^{-1}\right]}\end{array}$} \\
\hline $\mathrm{Em}+\mathrm{Ab}$ & 179 & 5186.2 & 54.3 & $727.0_{-35.8}^{+41.9}$ & $\ldots$ \\
$\mathrm{Em}$ & 42 & 4821.5 & 168.0 & $1088.8_{-103.0}^{+143.4}$ & $\ldots$ \\
$\mathrm{Ab} b_{137}$ & 137 & 5247.6 & 49.0 & $573.7_{-32.0}^{+38.3}$ & $601.5_{-37.6}^{+46.1}$ \\
$\mathrm{Ab}_{135}$ & 135 & 5256.6 & 46.7 & $542.9_{-30.5}^{+36.6}$ & $578.1_{-36.3}^{+44.5}$ \\
& & & & & \\
\hline
\end{tabular}

Note. - Velocity dispersions are computed from galaxies within $1.72 h^{-1}$ Mpcof the cluster center for Ab samples, and within 1.80 $h^{-1}$ Mpcfor Em and Em+Ab samples. $\sigma_{200}$ is the projected velocity dispersion within $r_{200}(\S 5.1)$.

Table 3. NFW $r_{c}$ from Projected Radii

\begin{tabular}{lcccc}
\hline \hline Sample & $r_{c} /\left[h^{-1} \mathrm{Mpc}\right]$ & $1 \sigma$ range & $2 \sigma$ range & $3 \sigma$ range \\
\hline $\mathrm{Ab}_{137}$ & 0.223 & $0.174-0.285$ & $0.157-0.316$ & $0.145-0.342$ \\
$\mathrm{Ab}_{135}$ & 0.211 & $0.164-0.270$ & $0.148-0.299$ & $0.137-0.324$ \\
& & & & \\
\hline
\end{tabular}

Note. $-r_{c}$ is computed from two-zone magnitude-limited samples of 123 and 121 members, respectively. 
Table 4. NFW Profile Parameters

\begin{tabular}{cccccccc}
\hline \hline Sample & $\begin{array}{c}r_{c} \\
{\left[h^{-1} \mathrm{Mpc}\right]}\end{array}$ & $\begin{array}{c}r_{200} \\
{\left[h^{-1} \mathrm{Mpc}\right]}\end{array}$ & $c$ & $\delta_{c}$ & $\begin{array}{c}r_{v} \\
{\left[h^{-1} \mathrm{Mpc}\right]}\end{array}$ & $\begin{array}{c}\mathrm{M}_{200} \\
{\left[10^{14} h^{-1} \mathrm{M}_{\odot}\right]}\end{array}$ & $\begin{array}{c}\mathrm{M}\left(<1.7 h^{-1} \mathrm{Mpc}\right) \\
{\left[10^{14} h^{-1} \mathrm{M}_{\odot}\right]}\end{array}$ \\
\hline $\mathrm{Ab}_{137}$ & 0.223 & 1.02 & 4.6 & $7.1 \times 10^{3}$ & 1.30 & 2.47 & 3.49 \\
$\mathrm{Ab}_{135}$ & 0.211 & 0.97 & 4.6 & $7.2 \times 10^{3}$ & 1.28 & 2.13 & 3.01 \\
& & & & & & \\
\hline
\end{tabular}

Note. $-r_{c}$ is computed from two-zone magnitude-limited samples of 123 and 121 members, respectively.

Table 5. NFW and Virial Masses

\begin{tabular}{lccc}
\hline \hline Sample & $\begin{array}{c}M_{\mathrm{NFW}}\left(<1.7 h^{-1} \mathrm{Mpc}\right) \\
{\left[10^{14} h^{-1} \mathrm{M}_{\odot}\right]}\end{array}$ & $\begin{array}{c}M_{\mathrm{VT}}\left(<1.7 h^{-1} \mathrm{Mpc}\right) \\
{\left[10^{14} h^{-1} \mathrm{M}_{\odot}\right]}\end{array}$ & $\Delta M / M_{\mathrm{VT}}$ \\
\hline $\mathrm{Ab}_{137}$ & 3.49 & 3.10 & 0.126 \\
$\mathrm{Ab}_{135}$ & 3.01 & 2.74 & 0.099 \\
\hline
\end{tabular}



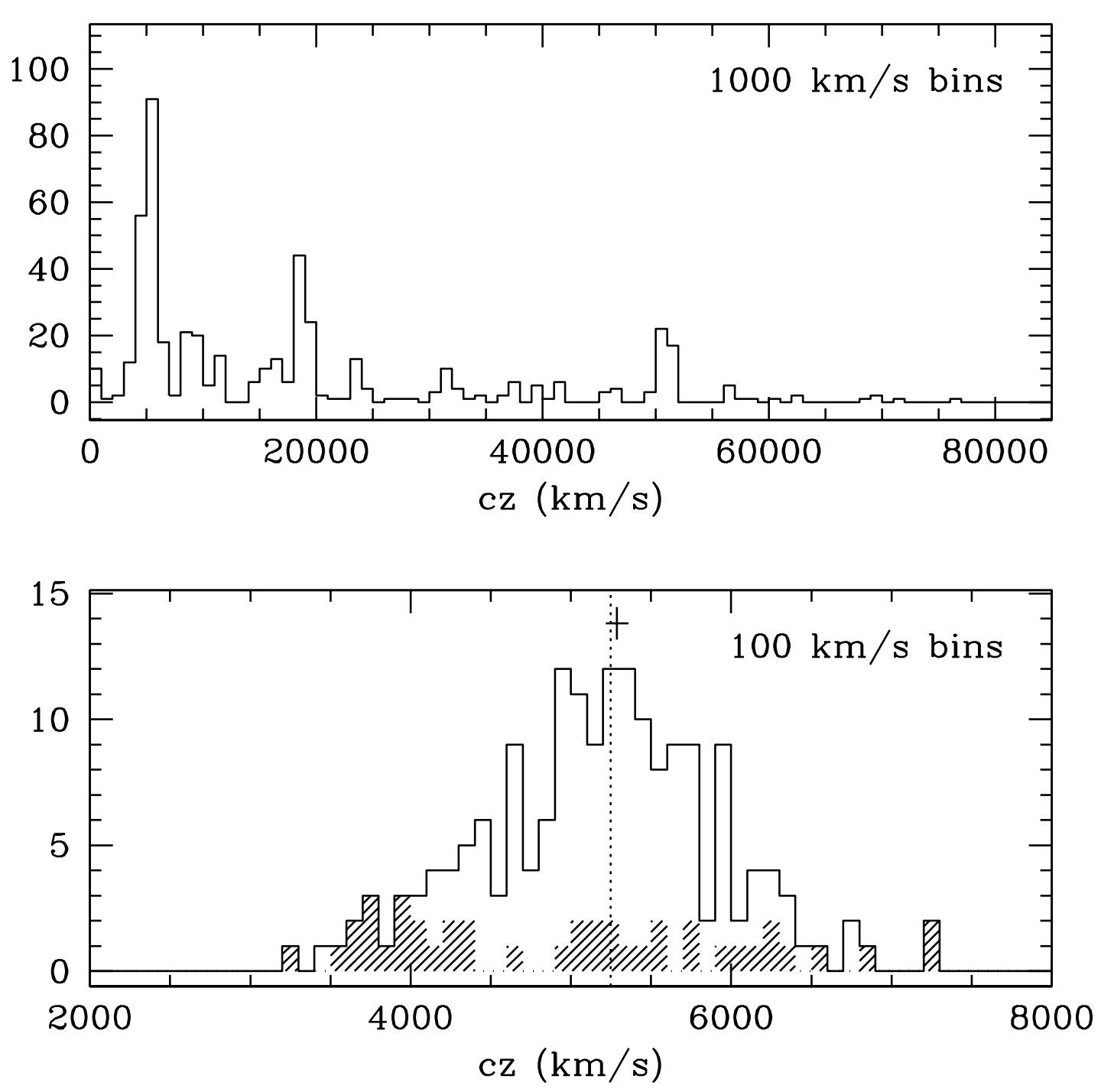

Fig. 1. - Top: Velocity histogram of all observed targets. Bottom: Velocity histogram of cluster members. The solid histogram denotes the entire sample (both Em and Ab galaxies); the shaded histogram denotes Em galaxies only. The dotted vertical rule indicates the mean redshift of the Ab galaxies; the solid vertical dash over the histogram indicates the redshift (and error) of the central cD galaxy. 


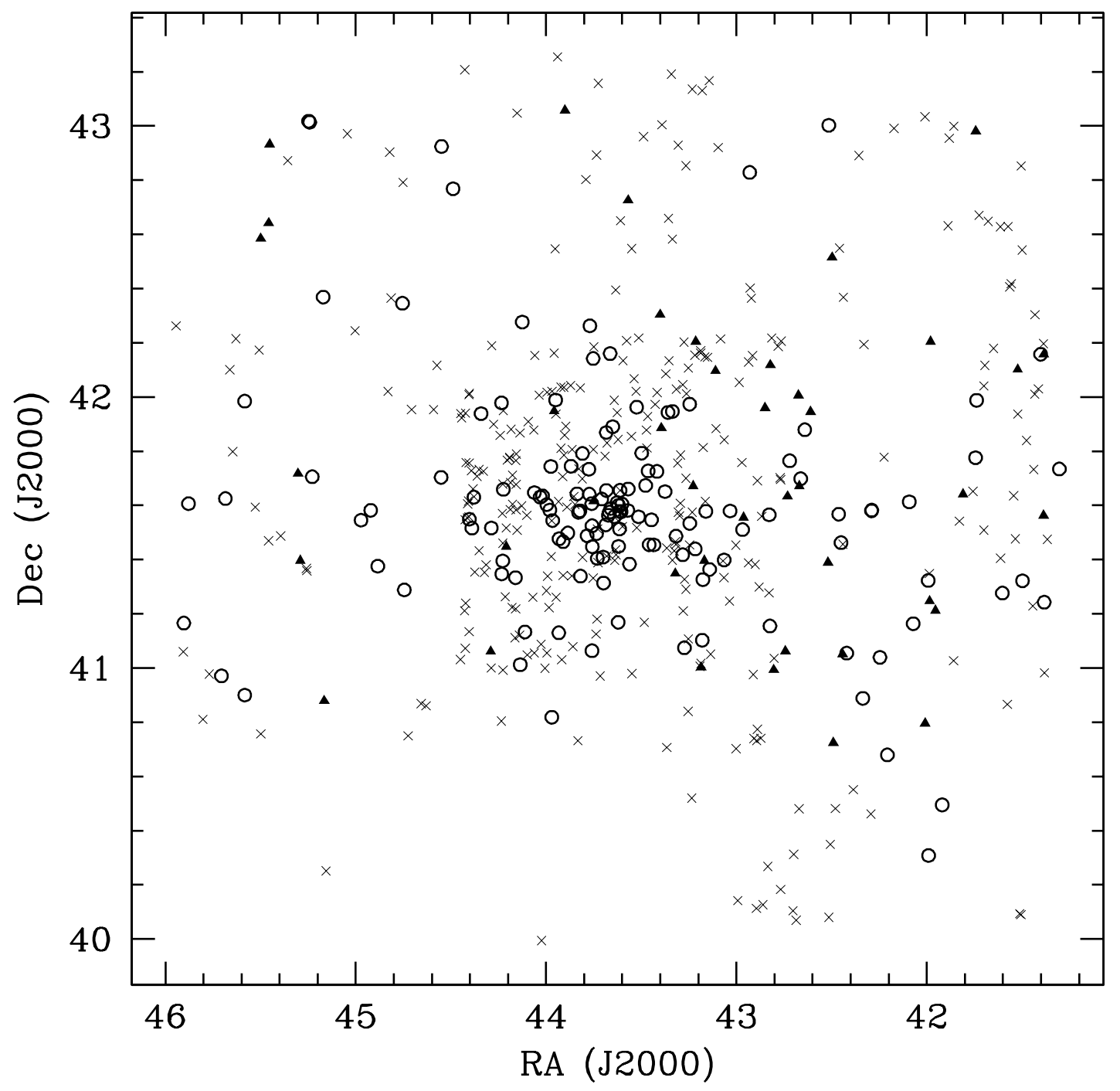

Fig. 2.- Position on the sky of all objects with measured redshifts. Note the denser sampling at the center, in the region covered by the Hydra and MMT observations. Crosses denote foreground and background objects. Open circles denote Ab galaxies in the cluster. Filled triangles denote Em galaxies in the cluster. Coordinate axes are in decimal degrees. 


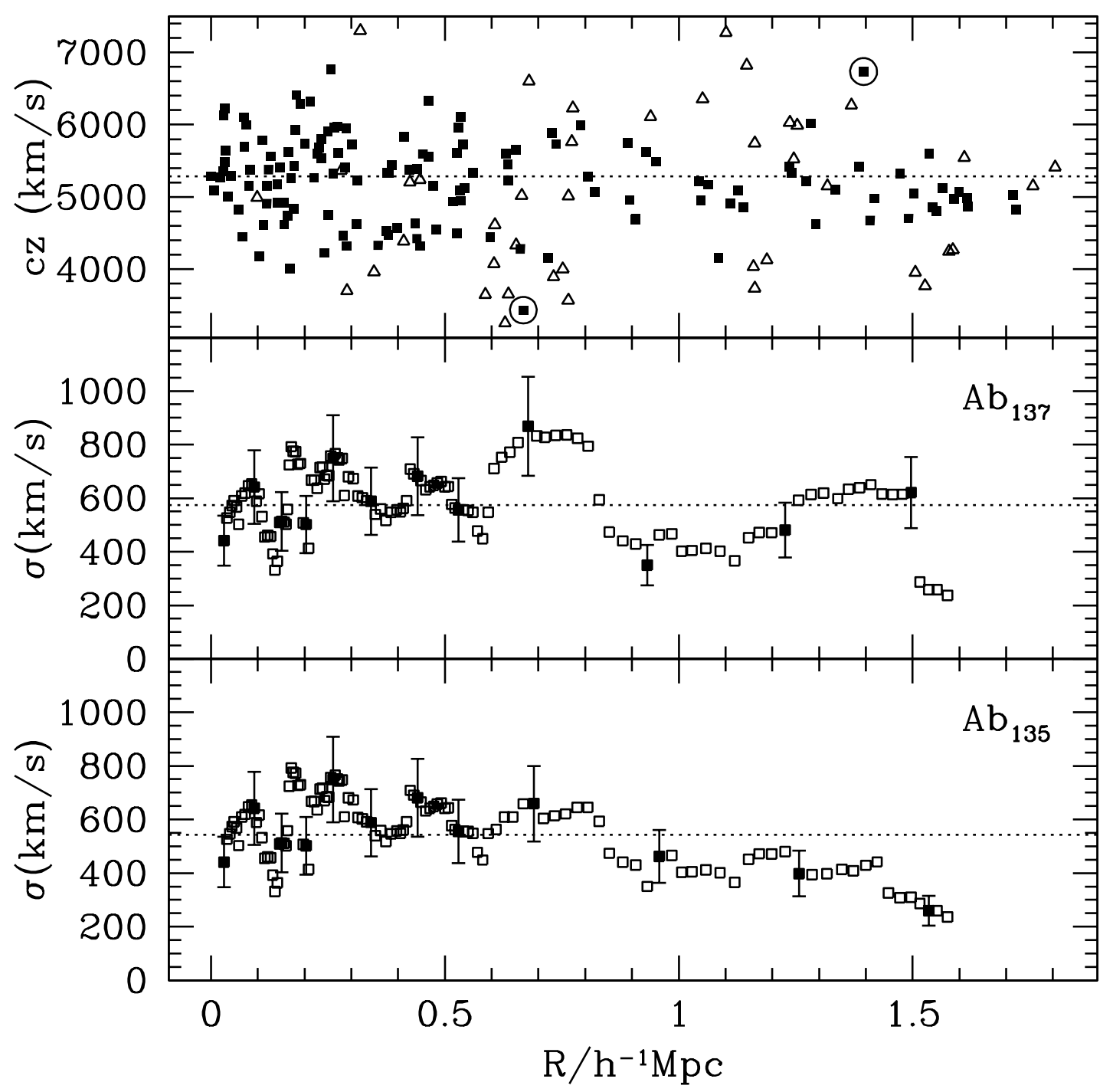

Fig. 3.- Top: Velocity as a function of projected distance from the cluster center. Ab galaxies are filled squares, Em galaxies are open triangles. The two outliers are circled. Middle: Velocity dispersion profile for the $137 \mathrm{Ab}$ galaxies in the cluster sample. Every eleventh point is independent and is shown with $1 \sigma$ error bars. The horizontal rule indicates these galaxies' overall dispersion of $574 \mathrm{~km} \mathrm{~s}^{-1}$. Bottom: Same as middle panel, but with two outliers removed. Horizontal rule is now at $543 \mathrm{~km} \mathrm{~s}^{-1}$. 


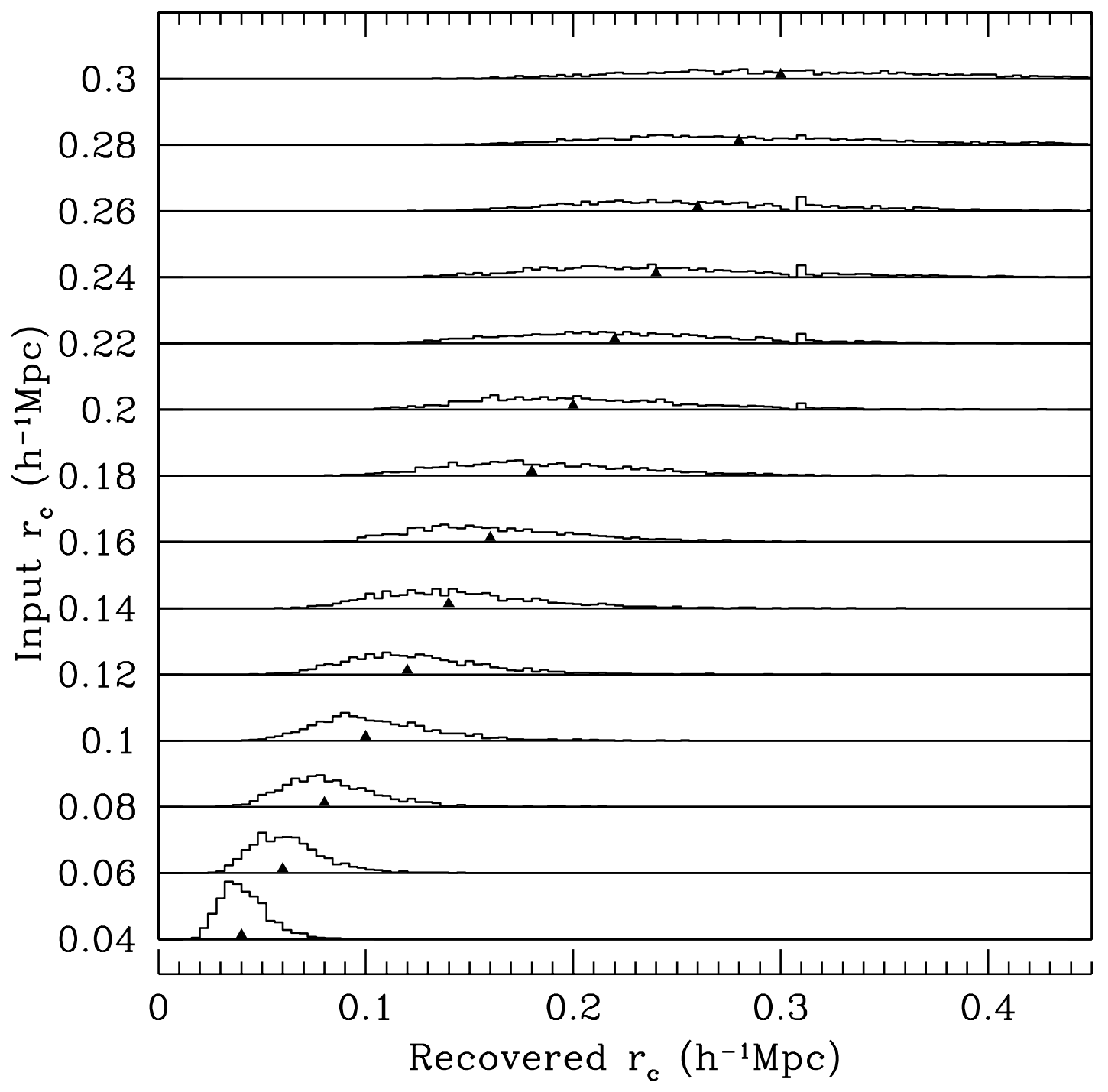

Fig. 4. - Histograms of recovered values of the NFW parameter $r_{c}$ from 2000 Monte Carlo realizations of sets of 123 projected radii drawn from NFW models with varying $r_{c}$. The histograms are all to the same scale, but for the highest input $r_{c}$ may extend off the right edge of the plot. The triangles in the histograms and the numbers along the vertical axis both indicate the input value of $r_{c}$. 

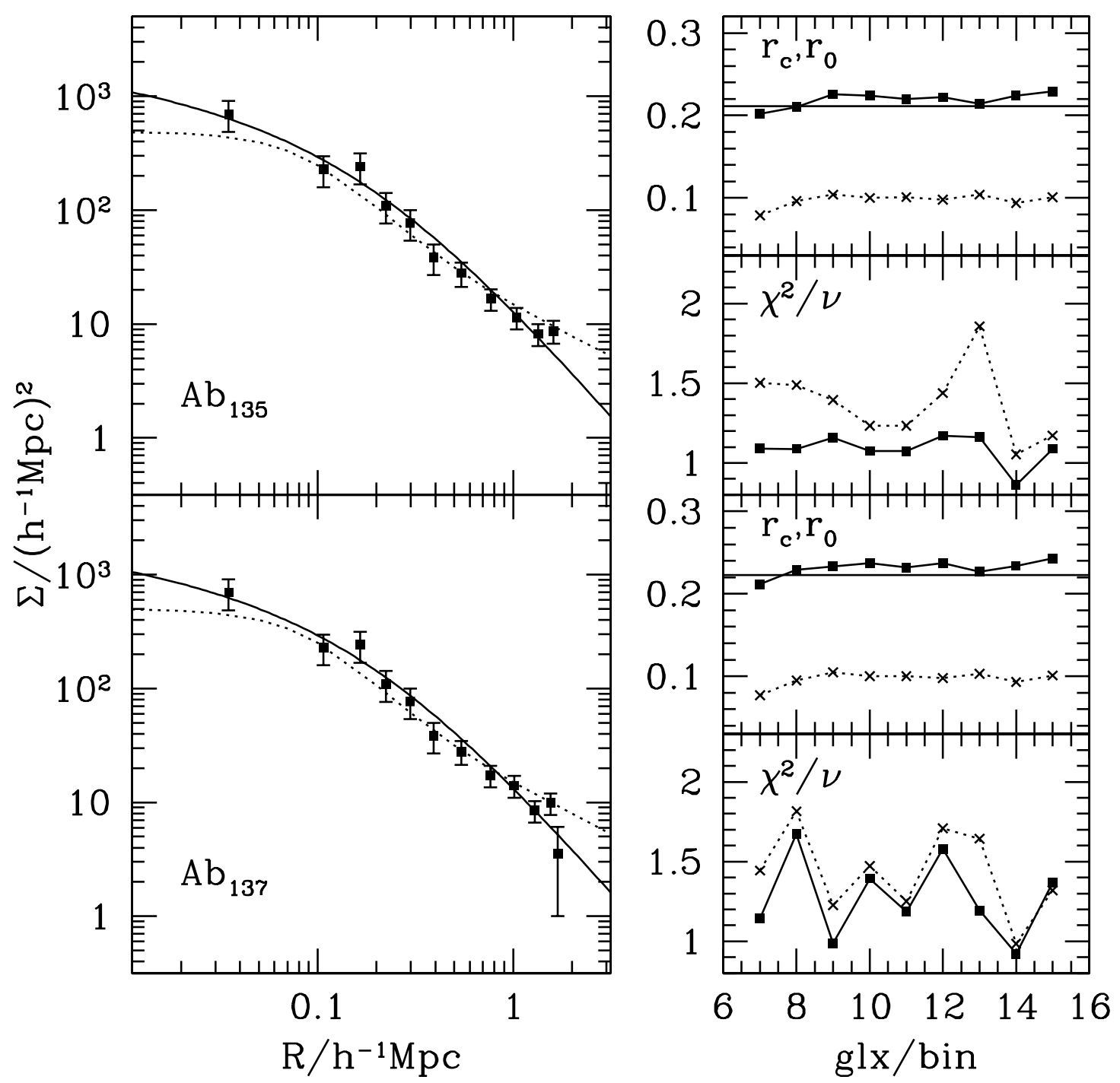

Fig. 5.- Comparison of the actual binned projected surface number density of galaxies to the NFW (solid) and non-singular isothermal sphere models (NSIS, dotted). The top half of the figure refers to the $\mathrm{Ab}_{135}$ sample; the bottom half to the $\mathrm{Ab}_{137}$ sample. Left: the actual surface density profile is shown as the dots with error bars; there are 11 galaxies per bin. The curves show the best-fit NFW and NSIS models. Right: dependence of the fits on the binning. The behavior of the best-fit value of $r_{c}$ (for the NFW model) or $r_{0}$ (for NSIS) and of $\chi^{2} / \nu$ is shown as a function of the binning. The horizontal rules indicate the maximum-likelihood values of $r_{c}$ for the two samples. 


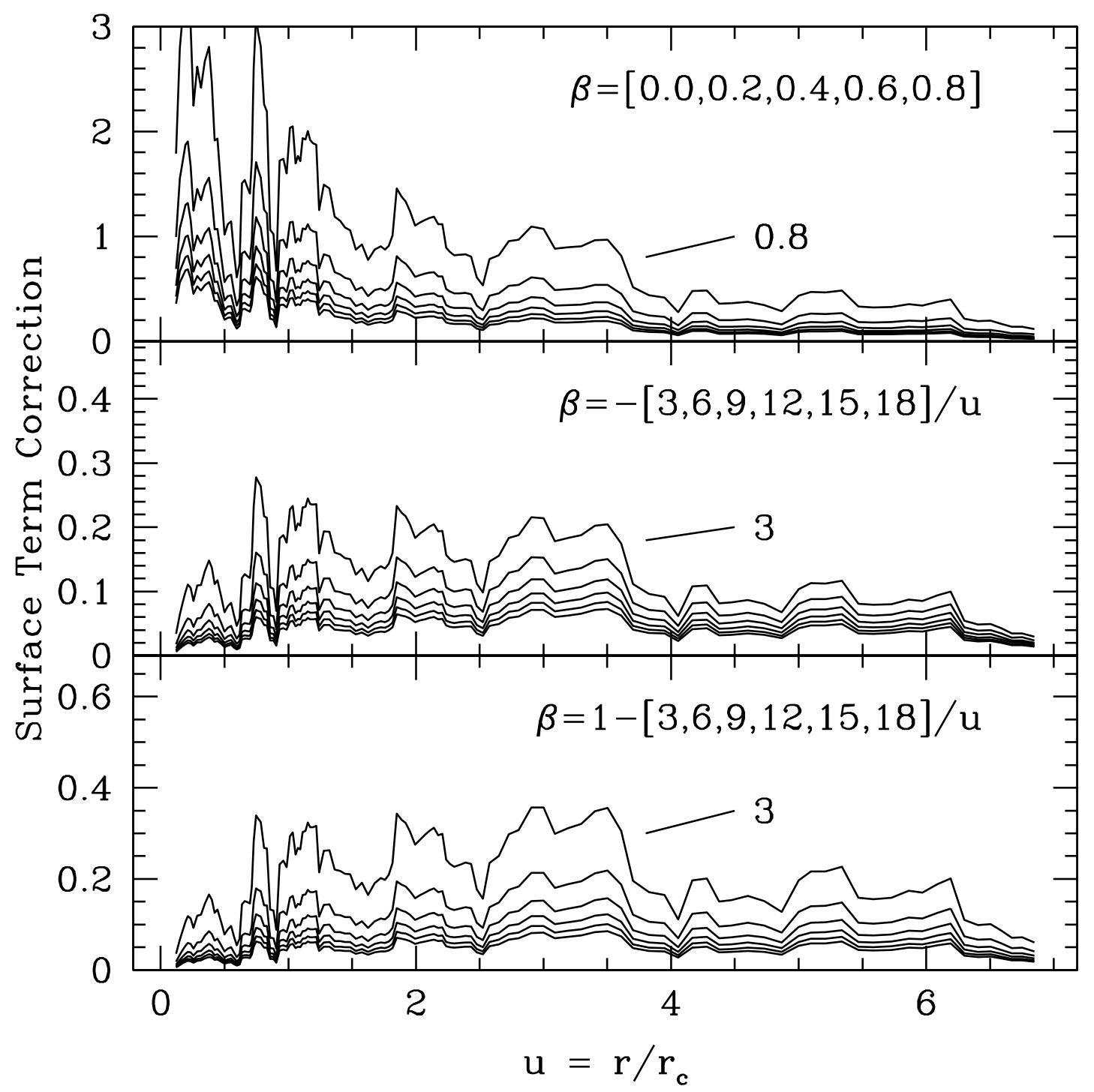

Fig. 6. - Behavior of the surface term correction to the virial mass estimator as given by Eq.10 assuming an underlying NFW mass profile for three models of the anisotropy profile $\beta(r)$. The assumed NFW profile uses $r_{c}=0.211$ (the best-fit value for the $\mathrm{Ab}_{135}$ sample), and we use the observed velocity dispersion profile. Top panel: $\beta(r)=k$; middle: $\beta(r)=-k / r$; bottom: $\beta(r)=1-k / r$, where $k$ is a constant in each case. There is one curve for each value of $k$ listed in the label, with one of the extreme curves labeled for reference. 


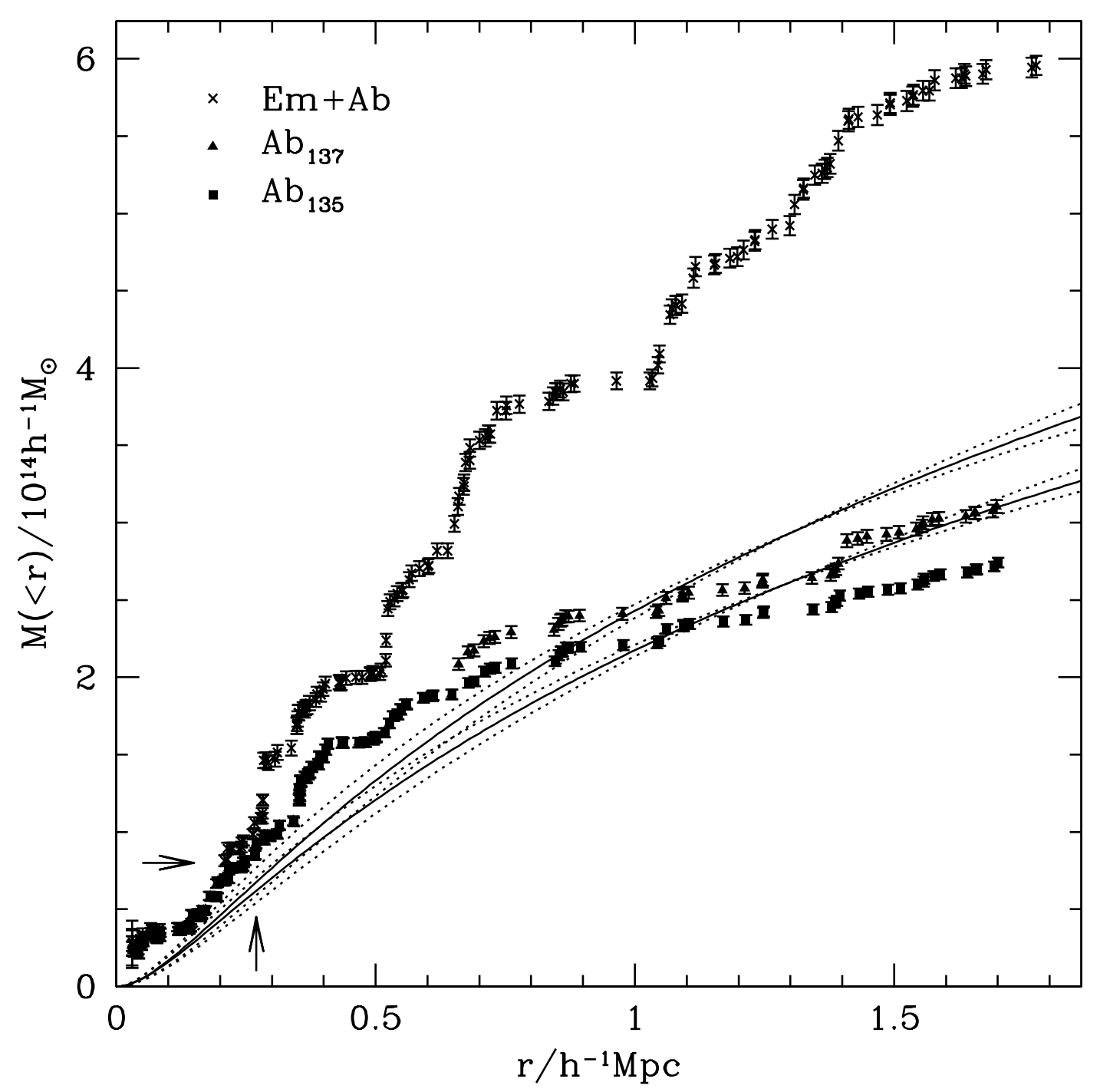

Fig. 7.- Enclosed mass profile as determined from the virial theorem, for Em, Ab $b_{137}$, and $\mathrm{Ab}_{135}$ samples. Error bars show the formal bootstrap errors (see text). The solid curves are the independently derived NFW profiles tabulated in Table 4 ; the dotted curves are the profiles corresponding to the $1 \sigma$ ranges of $r_{c}$ from Table 3 with $r_{200}$ recomputed from Eq. 7. The arrows point to the X-ray mass of Dell'Antonio et al. (1995). 